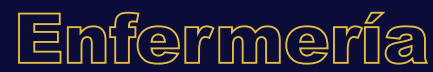

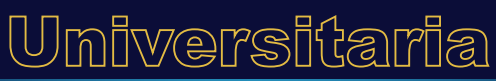

\section{Apariencia y masculinidad en enfermería: percepción de la vestimenta de enfermeros costarricenses}

\author{
Appearance and masculinity in \\ nursing: perception about the outfit \\ of male nurses in Costa Rica
}

\section{Aparência e masculinidade em enfermagem: percepção do vestuário de enfermeiros costa-riquenhos}

\author{
A. López-Badilla \\ ORCID: \\ ${ }^{\mathrm{a}}$ 0000-0002-4787-7411 \\ Universidad de Costa Rica, Escuela de Enfermería, San José, Costa Rica \\ Recibido: 12 marzo 2020
}

Aceptado: 23 febrero 2021

\section{RESUMEN}

Introducción: Históricamente, el uniforme blanco caracteriza a la profesión de enfermería. Hoy existe una amplia variedad de estilos vestimentarios que están transformando la percepción sobre la profesión y el cuidado enfermero; ello coincide con una mayor participación de varones donde interviene su organización e imagen social.

Objetivo: Analizar el rol de la apariencia en la construcción de la imagen social de la enfermería desde la percepción de los varones enfermeros sobre su vestimenta profesional.

Métodos: Entrevista a profundidad a ocho enfermeros costarricenses para comprender desde un marco interpretativo fenomenológico el significado de sus experiencias y vivencias en la profesión. Se realizaron inferencias interpretativas con base en marcos teóricos de 
enfermería y masculinidades que permitieron organizar dichas experiencias en unidades temáticas para este análisis de la vestimenta como referente de profesión marcado por el género.

Resultados: Se identificaron cuatro temáticas: las enfermeras visten de blanco, las variaciones en la vestimenta marcan jerarquías sociales, los varones agregan elementos en su vestido y, discurso que los identifica como enfermeros, esto con el fin de elaborar una imagen masculina de la enfermería que le otorgue mayor prestigio social.

Conclusiones: Los varones aceptan el uso del uniforme blanco, de color o estampado para los miembros subordinados; sin embargo, para los puestos altos y para los varones profesionales prefieren la ropa de vestir, pues creen que refuerza su estatus o posición, legitima el poder y la autoridad. En ese sentido, consideran que otorga una apariencia que rescatará a la profesión de su bajo estatus social.

Palabras clave: Imagen corporal; enfermería; masculinidad; vestuario; uniforme; investigación cualitativa; fenomenología; Costa Rica.

\section{ABSTRACT}

Introduction: Historically, the white uniform has characterized the nursing profession. Nevertheless, currently, there is a wide variety of nursing outfit styles which are transforming the perception about the profession, including aspects related to the organization and social image.

Objective: To analyze the impact of appearance in the construction of the social image of nursing from the perception of male nurses regarding their uniform.

Methods: An in-depth interview was conducted on 8 male nurses from Costa Rica in order, from an interpretative phenomenological frame, to better understand the meaning of the male nurses' lived experiences in the profession. These interpretative inferences based on nursing theoretical frames allowed an organization of the nurses' experiences into thematic units.

Results: Diverse themes emerged including: nurses dress in white; the variations in the nursing outfit denote social hierarchies; male nurse add elements to their outfits in order to be specifically identified and attributed with a masculine image of higher social prestige.

Conclusions: Male nurses accept the use of a white, or colored uniform for those subordinated staff; however, higher rank male nurses prefer wearing regular civil outfit, considering it as a reinforcement of their status or position of power and authority which should imply a higher social status.

Keywords: Body image; nursing; masculinity; clothing; uniform; qualitative research; phenomenology; Costa Rica.

\section{RESUMO}

Introdução: Historicamente, o uniforme branco caracteriza a profissão de enfermagem. Hoje existe una grande variedade de estilos de roupas que estão transformando a percepção da profissão e o cuidado de enfermagem; isso coincide com uma maior participação do homem onde intervém a sua organização e imagem social.

Objetivo: Analisar o papel da aparência na construção da imagem social da enfermagem a partir da percepção dos enfermeiros do sexo masculino sobre seu vestuário profissional. 
Métodos: Entrevista em profundidade com oito enfermeiros costa-riquenhos para compreender a partir de um marco interpretativo fenomenológico o significado de suas experiências e vivências na profissão. Realizaram-se inferências interpretativas a partir de marcos teóricos de enfermagem e masculinidades que permitiram organizar essas experiências em unidades temáticas para esta análise do vestuário como referente de profissão marcado pelo gênero.

Resultados: Identificaram-se quatro temáticas: as enfermeiras vestem-se de branco, as variações no vestuário marcam hierarquias sociais, homens acrescentam elementos em suas vestimentas e discursos que os identificam como enfermeiros, isto com a finalidade de formar uma imagem masculina da enfermagem que lhe confere maior prestígio social.

Conclusões: Os homens aceitam o uso do uniforme branco, colorido ou estampado para os membros subordinados; no entanto, para os cargos de chefia e para os homens profissionais preferem roupa formal, pois acreditam que isso reforça seu status ou posição, legitima o poder e a autoridade. Nesse sentido, consideram que dá uma aparência que resgatará a profissão de seu baixo status social.

Palavras chave: Imagem corporal; enfermagem; masculinidade; vestuário; uniforme; pesquisa qualitativa; fenomenologia; Costa Rica.

\section{INTRODUCCIÓN}

La preocupación sobre la apariencia corporal emerge tempranamente en el desarrollo de la enfermería. Parte de las propuestas de modernización del cuidado de Florence Nightingale abordaban la transformación de la imagen social de las enfermeras para mejorar la opinión pública sobre esta profesión. De igual manera, King ${ }^{1}$ reconoció su relevancia en el establecimiento de las relaciones terapéuticas entre las enfermeras y las personas usuarias al incluir en sus sistemas personal e interpersonal que el cuidado inicia con la percepción. En ese proceso las personas reaccionan a la apariencia de los otros, desarrollan expectativas y modifican sus conductas para lograr el establecimiento de transacciones eficaces. Las investigaciones demuestran que bastan sólo segundos de interacción para desencadenar complejas respuestas con consecuencias duraderas para la provisión de cuidados de enfermería ${ }^{2-4}$; en ellos la primera impresión generada por la vestimenta de la persona cuidadora es fundamental.

En ese sentido, Kalish y Kalish² advierten que la percepción del público sobre las habilidades, conocimientos y el profesionalismo del personal enfermero varían de acuerdo a cómo visten. Quienes defienden el uso del uniforme indican que éste permitirá proyectar una imagen clara y sin ambivalencias, discernible para la persona usuaria, quien reaccionará en correspondencia a lo que ha aprendido de la enfermería ${ }^{5-7}$.

El uniforme también ha sido concebido como un instrumento a través del cual se puede identificar el legado histórico de la profesión. Sobre ello se suele advertir, tanto por el personal de enfermería como el público, que el uniforme blanco refleja los valores profesionales de entrega, servicio, diligencia, disciplina y calidez, los cuales se asocian al cuidado3.7. Por otro lado, quienes usan uniformes coloridos o decorados con estampados llamativos suelen ser percibidos como menos profesionales, poco confiables y se les confunde con personal de otros equipos sanitarios y no sanitarios como nutrición, terapia respiratoria y conserjería ${ }^{8}$. 
La relación compleja, contradictoria, incluso paradójica de la enfermería con el público, entre sus propios miembros y con las otras profesiones 9 , se refleja en la diversidad de respuestas hacia los distintos patrones vestimentarios que actualmente son usados por enfermería. Al respecto, el énfasis del profesionalismo se caracteriza con cualidades distintas para varones y mujeres. El uso de estampados o colores llamativos caracterizan el profesionalismo como la capacidad para establecer relaciones afectivas, cercanas e íntimas, lo cual es típicamente incentivado en las mujeres enfermeras; mientras que en los varones el profesionalismo se expresa a través de los colores oscuros de la ropa formal, los cuales representan capacidades de gestión, liderazgo, racionalidad y éxito económico ${ }^{8}$. Hay quienes han señalado esto como un marcador de la trayectoria de los varones en la profesión ${ }^{10,11}$.

Si bien en Costa Rica no se cuenta con estudios directamente enfocados en los efectos de la vestimenta en el imaginario social de los usuarios con respecto a este sector profesional, el tema ha sido abordado superficialmente. En ellos se concluye que la sociedad distingue a la enfermería por su uniforme blanco, limita su lugar de práctica a los hospitales y la subordina a la autoridad médica. En el caso del país, el uniforme blanco en apariencia desvirtúa el profesionalismo de las enfermeras o, en esta misma línea, impide sean reconocidas como tales, lo cual resulta ambivalente ya que los subgrupos no profesionales suelen vestirse de forma similar a las enfermeras ${ }^{10}$.

La tendencia observada en Costa Rica de usar distintos patrones vestimentarios ofrece un importante campo de estudio aún por abordar. De igual manera, con la amplia incursión de los varones en esta disciplina el escenario plantea interrogantes en términos de cómo incide la percepción tradicional en su incorporación y mantenimiento, en las estrategias que este segmento ha implementado para resignificar los códigos tradicionales constitutivos de la imagen social de la profesional, así como negociar su masculinidad en un ámbito donde aún se promueven símbolos históricamente asociados a la feminidad.

Investigaciones con sustento en teorías feministas y de masculinidad han advertido la deuda pendiente de la disciplina gracias a la generación de conocimiento con perspectiva de género sobre la cotidianidad de la práctica enfermera ${ }^{1,2,13}$, Costa Rica no escapa a ese llamado. La percepción de la apariencia se aborda en este documento a través de los discursos de enfermeros varones sobre la vestimenta y la imagen social de su profesión. La imagen social existente acerca de la profesión actúa como sistema de valores, ideas y prácticas con la doble función de, por un lado, establecer un orden que facilite a los individuos distinguir las reglas de interacción; por el otro, proveer un código de comunicación inteligible que permite la realización del cuidado con el fin de conservar el orden ${ }^{3}$ establecido. Así, esta investigación indaga las percepciones de varones enfermeros sobre la apariencia para desentrañar el rol de la vestimenta en la configuración de la enfermería moderna y de la masculinidad en el cuidado.

\section{MÉTODO}

Se diseñó una investigación cualitativa de corte fenomenológico interpretativo en la que fueron analizadas las experiencias y vivencias del ejercicio de enfermería realizado por varones con el propósito de comprender su realidad social y los sentidos que orientan sus acciones ${ }^{14}$. Se tomó la vestimenta como dispositivo para expresar formas de organización social que intervienen en las interacciones sociales y genéricas ${ }^{12}$. Esto permite comprender la cotidianidad en el ejercicio de la enfermería y su efecto en quienes la practican, con lo cual se profundiza en la vivencia personal e interpersonal del cuidado ${ }^{15}$. En ese sentido, la narración de tales eventos no sólo ilustra el diario vivir de 
la profesión, además desentraña sus transformaciones, las cuales pueden ser extraídas en discursos como el de la vestimenta y la imagen social de la profesión para rastrear sus cambios.

Participaron 8 informantes con edades entre 25 y 6o, con experiencia profesional superior a 5 años, trabajadores en centros públicos del sistema de salud costarricense localizados en el Área Metropolitana, quienes fueron entrevistados entre septiembre de 2015 y enero de 2016. Fueron seleccionados primero por conveniencia y posteriormente por bola de nieve; para ello se tuvo comunicación con enfermeros que proveyeron contactos con posibles participantes, a quienes se les comentó vía telefónica los pormenores de su participación y se les presentó el formulario de consentimiento informado para aclarar los detalles de su colaboración. Dichas entrevistas fueron realizadas en espacios íntimos, sin interrupciones, previamente acordados entre el investigador y los participantes, con el fin de garantizar un adecuado flujo del diálogo para evitar la pérdida de información por distracciones.

La investigación comenzó con una etapa de clarificación de presupuestos, descrita como una profunda reflexión sobre las preconcepciones existentes en el autor que pudieran sesgar la recolección de la información ${ }^{16}$. Para ello, se inició la revisión de antecedentes de estudios similares y la clarificación de los conceptos teóricos con los cuales se analizaría la información. La siguiente etapa, llamada descriptiva, inició con la formulación de la guía de entrevista y culminó con la elaboración de la descripción protocolar, es decir, con la transcripción de las entrevistas y las anotaciones iniciales ${ }^{17}$.

Posteriormente, se aplicó el método de triangulación expuesto por Cisterna ${ }^{16}$ para ordenar conceptual y temáticamente los discursos, lo que dio inicio a la etapa descriptiva con la revisión final de las transcripciones y concluyó con la discusión de resultados. En esta etapa, conforme los discursos alcanzaron su nivel de saturación, es decir, dejaron de aportar nueva información y agotaron los temas al tornarse recurrentes, fueron agrupados como inferencias interpretativas con conclusiones de primer orden (conceptos del Sistema de King ${ }^{1}$ como son: percepción, yo, imagen corporal, rol, comunicación, estatus, poder), segundo orden (personal, interpersonal y social) y de tercer orden (un resumen general de los discursos).

Se agregó una última etapa del análisis, correspondiente a la triangulación teórica, la cual se realizó a partir de los planteamientos teóricos de Connel118,19 sobre las masculinidades. Se contrastó la información con las estructuras y relaciones de masculinidades propuestas en la concepción relacional del género de Connell y otros autores ${ }^{20,21}$, lo que permitió transformar las categorías apriorísticas en categorías analíticas emergentes. El proceso resultó en un discurso sobre la vestimenta como dispositivo para negociar la masculinidad en la enfermería.

\section{Consideraciones éticas}

La investigación fuente de estos relatos fue aprobada por el Comité Ético Científico de la Universidad de Costa Rica; además, estuvo supervisada por un comité asesor profesional aprobado por la Comisión de Trabajos Finales de Graduación de la Escuela de Enfermería de esa universidad, y recurrió a la elaboración de un Consentimiento Informado debidamente aprobado por el comité antes mencionado. Tales requisitos acompañaron las medidas para la protección de la identidad de los participantes y garantizaron el cumplimiento de pautas éticas para el tratamiento de la información y de los informantes.

En dicho artículo se utilizan pseudónimos para nombrar a los participantes. Este texto cumple con el compromiso ético de devolver los beneficios del conocimiento construido. 


\section{RESULTADOS}

La imagen social de la enfermería prevaleciente corresponde a la enfermera vestida de blanco; sin embargo, existen prácticas y discursos que pretenden reformular esa imagen para legitimar la presencia de los varones en la profesión, situarlos en las posiciones superiores y legitimarlos en esos sitios. Esto puede rastrearse en la jerarquización reflejada en la vestimenta, la cual muestra una importante relación con una perspectiva de género patriarcal.

\section{Las enfermeras visten de blanco}

El personal de salud se reconoce y jerarquiza conforme a su apariencia y género. Mientras la medicina persiste como profesión de varones distinguida por su vestimenta formal; la enfermería se concibe como profesión de mujeres y es identificada por el uniforme blanco principalmente, al cual se suman elementos distintivos que expresan el estatus social y profesional:

Mira cómo la imagen concreta muchas veces la profesión, y nuestra profesión tiene ya su prototipo. Usted sabe que la enfermera es la que tiene la cofia, es la que viste de blanco, entonces de ahí que confundan al auxiliar con la enfermera. Entonces ves que todo es una percepción de imagen (Fabricio).

\section{La vestimenta expresa jerarquías sociales}

Las variaciones vestimentarias expresan jerarquías sociales vinculadas al género, la sexualidad y el poder. El uso del uniforme blanco está destinado a las mujeres y los puestos inferiores; la vestimenta formal es para los varones, quienes ejercen puestos superiores. El vestir de blanco implica que el varón se cuestione su virilidad.

No sé por qué me hizo sentir incómodo, tal vez porque era más chamaco. Pero igual todavía no me gusta el uniforme de enfermería, que sea zapato blanco, zapatilla blanca y tal vez sea por, igual, por estereotipo que tenga uno mismo. Pero igual es cuestión de gusto, eso no quiere decir que por usar un pantalón blanco no te haga masculino. Siento que no es atractivo ver a un man de blanco; al contrario, ver a una muchacha toda de blanco se ve muy bien (Mauro).

Mientras tanto, el uso de ropa formal refuerza elementos del imaginario tradicional de la masculinidad y promueve un mejor estatus para los varones:

Cuando a mí me ascendieron, recuerdo que la directora me llamó casi que el día antes, y de las cosas que ella me dijo se me clavaron dos: lo primero que me dijo: jtiene que venir con corbata! (...) y la segunda cosa que se me grabó fue: iyo espero de usted liderazgo! (Álvaro).

Los otros patrones de vestimenta se insertan en la jerarquía con el fin de consolidar la diferenciación del trabajo, de ahí que ocupen sitios intermedios con gradiente de género:

Como enfermería es un grupo: asistente, auxiliar, secretaria, enfermero, entonces ellos creen que todos tienen iguales derechos, iguales atributos, y hasta quisieran tener los mismos salarios, pero no quieren asumir responsabilidades (...). Eso suena como que yo soy muy orgulloso, pero me parece que es un conjunto, cada quien tiene que identificarse en lo que hace y eso es lo que cuesta (Ernesto).

\section{Identificarse como enfermero}

Con el cambio del patrón de vestimenta el enfermero es instado a reconocerse como profesional; sin embargo, puesto que el referente de identidad profesional masculino en enfermería es prácticamente inexistente para el público, el varón pasa a ser reconocido o confundido con un médico. Este efecto paradójico del ser y no parecer enfermero se percibe como una invalidación de la profesión, que implica la pérdida del reconocimiento social: 
Nosotros los enfermeros, si vestimos en los hospitales con corbata, camisa de manga larga, nos ponemos la gabacha y vestimos formalmente, los pacientes y los familiares nos dicen «doctor» (Fabricio). El enfermero, aunque fuera de blanco y estuviese bien, los pacientes refieren: «es que la enfermera es ella», $y$ «el médico él», y a las mujeres, podría ser la doctora y le decían enfermera (César).

\section{Legitimación de la masculinidad en enfermería}

Los informantes consideran que el reconocimiento que obtiene la enfermería a través de la mujer uniformada de blanco mantiene su estatus social inferior. De esta manera, la vestimenta se toma como recurso para impulsar un mayor reconocimiento social a través de la creación de una imagen social del enfermero que actuaría en el rescate de la profesión:

Estamos con un pensamiento muy corto todavia en el país. Enfermería está en una posición que nosotros mismos los profesionales hemos permitido porque no permitimos que se genere un cambio, nos falta mucho para tener un estatus diferente porque como gremio incluso no hemos llegado a un consenso, y todavía se ve al enfermero y la enfermera como el que viste de blanco y usa una cofia (Jairo).

Esto sostiene la intención de mantener las diferencias en la apariencia, que por un lado identificaría al personal de enfermería, y por el otro, así como ha ocurrido con los distintivos agregados a la cofia, permitiría reconocer la jerarquía de género configurada a través del enfermero masculino.

Y eso es otra cosa que yo a mi gabacha le pongo ENFERMERO. Mis iniciales y «enfermero». Yo nunca he querido que me comparen con un médico. ¿Por qué? ¡Porque el médico no hace lo que yo hago! ¡Ni yo hago lo que hace el médico! (Santiago).

\section{DISCUSIÓN}

Un componente del proceso de percibir es la categorización, que consiste en organizar los estímulos a partir de concepciones culturales y experiencias cotidianas. La apariencia en la vestimenta se categoriza de acuerdo con el contexto de uso; en ese sentido, las prendas utilizadas tienen la capacidad de comunicar significados, los cuales varían según el sitio y momento en que se llevan puestas ${ }^{12,22}$. La imagen social de la enfermería en Costa Rica vincula apariencia y contexto en la percepción, son enfermeras aquellas mujeres uniformadas de blanco, trabajadoras en hospitales y clínicas bajo la supervisión directa del médico ${ }^{10}$, quien es varón, viste ropa de calle y ejerce la máxima autoridad entre las profesiones de la salud ${ }^{23}$.

Estas dos construcciones constituyen la forma de percibir a los varones y las mujeres como personal sanitario, mismo orden que se refleja en el discurso sobre la reacción y los comentarios del público hacia quienes ejercen la enfermería. Por ende, aún persiste la idea, al menos en el imaginario social, de que las profesiones son específicas al sexo, ello constituye, a su vez, el contexto en el cual los varones negocian la masculinidad a través de la reconstrucción de los parámetros de categorización profesional.

El poder de la vestimenta para organizar las relaciones sociales se muestra en el gradiente social que subordina el uniforme blanco a los coloridos y estos a la ropa de calle. El profundo análisis realizado por Retana ${ }^{12}$ sobre la genealogía de la moda advierte al respecto que la vestimenta lleva a cabo mecanismos de control social a través de sistemas de marcación originados en el género dicotómico como principio de organización. Estas biopolíticas codifican a través del vestido las formas de subjetivación de los individuos, sus relaciones con los otros dentro del territorio, y establecen una correspondencia entre sexualidad, deseo, género y posición social. Así, la vestimenta participa en 
la definición del yo, de la identificación profesional y de la modelación performativa que define las prácticas y acciones a través de las cuales se presenta como enfermerog,24.

Al respecto, Retana ${ }^{12}$ aplica lo que Butler entiende por performatividad; es decir, una reiteración ritual de ciertas condiciones de prohibición y tabú que revelan un compromiso de reconocimiento con determinadas categorías aparentemente estables como el género. De esta manera, la apariencia que se pretende desplegar con la vestimenta se ajusta al acuerdo social de cómo debe verse un hombre o una mujer, pero también lo es con respecto a su ocupación, a la correspondencia entre trabajo y género.

En este aspecto, para los informantes el uniforme blanco socava doblemente la posibilidad de los enfermeros de ver satisfecha su masculinidad. Por un lado, porque conlleva la identificación con lo femenino; por el otro, implica asumir una masculinidad subordinada. En ese sentido, el abierto rechazo expresado en el discurso hacia el uniforme tradicional es un intento de expulsar de la apariencia cualquier aspecto que se identifique como femenino. Conviene advertir sobre el uso del uniforme blanco fuera del contexto hospitalario, el cual se aborda como un cuestionamiento de la heterosexualidad. Al respecto, Retana ${ }^{12}$ señala que la vestimenta expresa una jerarquía somática de correspondencia entre sexo, sexualidad, cuerpo, y deseo.

El uso de prendas como la gabacha, la corbata, adornos como el estetoscopio o los uniformes de colores oscuros preparan a los varones para interacciones sociales en las que se pone en evidencia su conocimiento y superioridad. Resaltan las campañas que promueven la incorporación de hombres enfermeros en las que se suele retratarlos con uniformes de colores sólidos oscuros, no de traje y corbata, esto con la finalidad de reforzar la hombría a través de aparatos e instrumentos tecnológicos $8,9,25$.

Entre las finalidades de la vestimenta se cita además una de las pretendidas ventajas de uniformar la apariencia. En términos de los referentes de identificación, una imagen homogénea cohesiona los grupos y crea pertenencia de tal manera que para el público es más fácil saber quién es el enfermero o la enfermera; es decir, visibiliza su trabajo, con lo cual obtiene el reconocimiento social que se busca. La intención de las personas al uniformar su apariencia con un grupo determinado es comunicarle que comparte sus valores, creencias y prácticas, con ello procura integrarse en ese colectivo. Así, la vestimenta cumple su función performativa de compromiso con la identidad del grupo y se convierte en un vehículo para integrarse a uno previamente conformado. Sin embargo, en tanto esa identidad está traspasada por el régimen de género puede resultar paradójica, tal como ocurre cuando la apariencia corporal no coincide con la expectativa social.

Retana $^{12}$ señala al respecto que, si bien la vestimenta tiene como meta, por un lado, identificar los grupos y al vestirse respectivamente los individuos actualizan su pertenencia a ellos; por el otro, muestra diferencias que procuran ordenar las jerarquías internas. Por consiguiente, como lo advierte Connell ${ }^{19}$, el sistema genérico suele recurrir a mecanismos simbólicos para reafirmar el ordenamiento social. Así, los colores y los estampados refieren al sexo, el género y la sexualidad, preservan jerarquías, recrean los parámetros de recognoscibilidad que adscriben no sólo a la profesión, sino al sexo de quien porta la prenda.

El aspecto más discutido en torno a las características que atribuye la vestimenta aborda la percepción de los distintos conocimientos necesarios en la enfermería. Sobre esto, el uso de uniformes con colores oscuros se asocia con mayor confianza, dinamismo, agresividad y decisión; mientras que los diseños animados suelen transmitir calidez, accesibilidad o empatía. Las tendencias específicas al sexo muestran que los colores oscuros son vestidos por varones, mientras que los diseños 
animados son utilizados por mujeres. Los efectos en la forma en que estos patrones vestimentarios tienen en la promoción laboral se han comprobado ${ }^{26}$ y constituyen elementos de diferenciación del profesionalismo en relación con el sexo del personal de enfermería.

Por otra parte, la vestimenta, en tanto dispositivo ceremonial también puede entenderse a través del poder que se le ha conferido para jerarquizar; ejemplo de ello son los listones agregados a la cofia, la adhesión de cualquier otro fetiche que, en términos de Foucault citado por Retana ${ }^{12}$, afianza rasgos identitarios mediante signos corpóreos. La metamorfosis del enfermero ascendido al que se le demanda autoridad y el uso de corbata refleja las palabras de Woolf al señalar la capacidad de la prenda para actualizar poderes y conductas como lo refiere Retana ${ }^{12}$. Dicha transformación constituye una ceremonia corporal que modifica la forma en la cual el enfermero se relaciona con su personal, ello es patente en lo explícito de solicitar autoridad, es decir, de hacerse reconocer con poder.

Preservar las diferencias de los patrones vestimentarios en relación con el sexo y el estatus del personal enfermero se convierte en una necesidad fundamental para mantener una jerarquía de diferenciación que marque la senda a seguir por los varones. En el discurso de los informantes se identifica la demanda que pretende que los subgrupos de enfermería inferiores en la jerarquía de clases laborales no sólo vistan con un patrón vestimentario correspondiente al subgrupo al que pertenecen, sino que también se espera actúen en concordancia con el puesto que poseen. Esto da sentido a la necesidad de mantener y ordenar apariencias para comunicar la posición subordinada; es decir, la relación que se tiene con los otros en el gremio.

Sin embargo, como se puede percibir, la ceremonia de metamorfosis con la que se patenta el estatus de un varón no ya como auxiliar de enfermería, sino como enfermero profesional -tal como se describió en la demanda planteada por una supervisora a uno de los informantes al ascenderlopuede ser paradójica en tanto el cambio de apariencia implica desligarse de la imagen social de la enfermería. En otras palabras, al vestirse de traje, ataviarse de gabacha, emular conductas de liderazgo y autoridad, el recién ascendido enfermero pasa a ser identificado como un médico.

Por último, los sistemas policiales de clasificación que marcan los cuerpos y les asignan su identidad distribuyen también las actividades a cada uno según su posición; Retana ${ }^{12}$ toma esto último como un aspecto de la vestimenta para marcar una relación con el espacio. Partiendo de que el espacio no es ajeno a las configuraciones de género ${ }^{12,19}$, se sigue que la distribución generalizada de los cuerpos en él contribuyen a legitimar instancias de subjetivación en tanto el acceso y las prohibiciones influyen en la percepción de sí mismo. Al respecto, la vestimenta, por identificar relacionalmente a su portador, autoriza sus desplazamientos y así permite contactos. El autor explica sobre este dispositivo que se despliega en dos mecanismos principales. Por un lado, el emplazamiento, que refiere según Foucault a las relaciones de vecindad, es decir, con quienes se tiene interacción inmediatamente; por el otro, el desplazamiento, que implica el movimiento entre diferentes espacios.

\section{CONCLUSIONES}

Desde la percepción de los participantes sobre la vestimenta se advierte que el uso del uniforme blanco constituye un factor que vulnera la masculinidad de los enfermeros puesto que implica investirse con referentes de identificación de feminidad. De igual modo, el uso de uniformes sitúa a quienes los visten en las posiciones sociales inferiores, también implican para ellos un cuestionamiento de su alcance de la masculinidad exitosa, hegemónica. Tanto los varones como las mujeres enfermeras han legitimado prácticas y discursos que mantienen esta percepción, lo cual se expresa en los discursos de diferenciación vestimentaria de los varones con respecto a las mujeres y a otros 
grupos no profesionales de enfermería. Desde la percepción de los varones, visibilizar al enfermero vestido de traje conllevará a un aumento en el estatus social de la profesión. Sin embargo, al no existir una imagen social del enfermero es necesario crearla y legitimarla para garantizar la meta antes mencionada.

Este informe de investigación forma parte de la primera aproximación directamente relacionada con masculinidad y varones profesionales en enfermería; su principal alcance lo constituye la teórica de masculinidades como vía para conocer las experiencias que viven los enfermeros como profesionales del cuidado comprendiéndolo desde sus propias voces.

Como limitaciones de este estudio se reconoce el bajo número de informantes, lo cual obedeció a la falta de interés en participar de los enfermeros contactados. Asimismo, es necesario incorporar la opinión de las mujeres enfermeras para profundizar en la percepción que tienen sobre las modificaciones vestimentarias. Hacen falta también estudios relacionados con la opinión del público acerca de la apariencia de las enfermeras y los enfermeros.

Finalmente, el estado actual del conocimiento sobre el tema de la vestimenta y la imagen profesional de la enfermería en Costa Rica es una fuerte limitante para alcanzar una discusión profunda que permita entender cómo se ha transformado la imagen de la enfermería en el país. Este estudio, además, se realiza con abordaje desde teorías de masculinidad, por lo que se requiere un enriquecimiento mediante el pensamiento feminista que permita ampliar la capacidad analítica de los fenómenos tratados.

\section{RESPONSABILIDADES ÉTICAS}

Protección de personas y animales. El autor declara que no se han realizado experimentos en personas ni animales.

Confidencialidad. El autor declara que se han seguido los protocolos necesarios para la protección de los datos de los informantes.

Derecho a la privacidad y consentimiento informado. El autor declara que en este artículo no aparecen datos sobre los informantes.

Conflicto de intereses. El autor declara no tener conflictos de intereses.

Financiamiento. Ninguno.

\section{REFERENCIAS}

1. King IM. Enfermería como profesión: filosofía, principios y objetivos. México: Limusa; 1984.

2. Kalisch B, Kalisch P. Dressing for success. Am J Nurs. 1985; 85(8): 887-93. https://bit.ly/3hHirc9

3. Skorupski VJ, Rea RE. Patients' perceptions of today's nursing attire. J Nurs Adm. 2006; 36(9): 393-401.

https://doi.org/10.1097/00005110-200609000-00005

4. Lennon SJ, Davis LL. Clothing and human behavior from a social cognitive framework part I: Theoretical perspectives. Cloth Text Res J.1989; 7(4):41-8.https://doi.org/10.1177/0887302X8900700406

5. Clavelle JT, Goodwin M, Tivis LJ. Nursing professional attire. J Nurs Adm. 2013; 43(3): 172-7. https://doi.org/10.1097/NNA.obo13e318283dc78

6. Timmons S, East L. Uniforms, status and professional boundaries in hospital. Sociol Health Illn. 2011; 33(7): 1035-49. https://doi.org/10.1111/j.1467-9566.2011.01357.x

7. León-Román CA. El uniforme y su influencia en la imagen social. Rev Cubana Enfermer. 2006; 22(1): 1-6. https://bit.ly/3fBbTJG 
8. Miller T, Mann N, Grim RD. Clothes encounter: Patient perception of nursing attire in a behavioral health unit. J Am Psychiatr Nurses Assoc. 2010; 16(3): 178-83.

https://doi.org/10.1177/1078390310370621

9. Thomas CM, Ehret A, Ellis B, Colon-Shoop S, Linton J, Metz S. Perception of nurse caring, skills, and knowledge based on appearance. J Nurs Adm. 2010; 40(11): 489-97.

https://doi.org/10.1097/NNA.obo13e3181f88b48

10. Solano-López AL. La imagen social de la enfermería en Costa Rica y su construcción desde la autoimagen profesional. Enferm. actual Costa Rica. 2012; 33(2): 54-6. https://bit.ly/3hImOUk

11. Alvarado-Quijano G, Alvarado-Rodríguez E, Barboza-Picado A, Castro-González É, León-Morales S, Matarrita-Matarrita A, et al. Definición de enfermería en el tercer nivel de atención según los acompañantes de los usuarios de un hospital costarricense. Enferm. actual Costa Rica. 2008; (14):1-10. https://bit.ly/2OHuY4w

12. Retana C. Las artimañas de la moda: una genealogía del poder vestimentario. San José, Costa Rica: Arlekín; 2015.

13. Polit DF, Beck CT. Nursing research: Generating and assessing evidence for nursing practice. 9th ed. Philadelphia: Lippincot Williams \& Wilkins; 2012.

14. Packer MJ. La ciencia de la investigación cualitativa. Bogotá: Uniandes; 2013.

15. Tuohy D, Cooney A, Dowling M, Murphy K, Sixsmith J. An overview of interpretive phenomenology as a research methodology. Nurse Res. 2013; 20(6): 17-20. https://ng.cl/18lmf

16. Cisterna-Cabrera F. Categorización y triangulación como procesos de validación del conocimiento en investigación cualitativa. Theoria. 2005; 14(1): 61-71. https://bit.ly/3vbfO6C

17. Martínez-Miguelez M. Comportamiento Humano. Nuevos mátodos de investigación. $2^{\mathrm{a}}$ ed. México: Trillas; 1996.

18. Connell RW. Masculinities. 2nd ed. California: University of California Press; 2005.

19. Connell R, Pearse R. Género desde una perspectiva global. Valencia: Universitat de València; 2018.

20. Connell RW, Messerschmidt JW. Masculinidade hegemônica: Repensando o conceito. Rev. Estud. Fem. 2013; 21(1): 241-82. https://doi.org/10.1590/S0104-026X2013000100014

21. Messerschmidt JW. Engendering gendered knowledge: Assessing the academic appropriation of hegemonic masculinity. Men Masc. 2012; 15(1): 56-76. https://doi.org/10.1177/1097184X11428384

22. Tennent E. Book Reviews. The fashioned body: Fashion, dress \& modern social theory Joanne Entwistle. Fem Psychol. 2018; 28(2): 292-306. https://doi.org/10.1177/0959353516682662

23. Ruetzler T, Taylor J, Reynolds D, Baker W, Killen C. What is professional attire today? A conjoint analysis of personal presentation attributes. Int J Hosp Manag. 2012; 31(3): 937-43.

https://doi.org/10.1016/j.ijhm.2011.11.001

24. Petrilli CM, Mack M, Petrilli JJ, Hickner A, Saint S, Chopra V. Understanding the role of physician attire on patient perceptions: A systematic review of the literature-targeting attire to improve likelihood of rapport (TAILOR) investigators. BMJ Open. 2015; 5(1): 1-18.

https://doi.org/10.1136/bmjopen-2014-006578

25. Clow KA, Ricciardelli R, Bartfay WJ. Are you man enough to be a nurse? The impact of ambivalent sexism and role congruity on perceptions of men and women in nursing advertisements. Sex Roles. 2015; 72: 363-76. https://doi.org/10.1007/s11199-014-0418-o

26. Porr C, Dawe D, Lewis N, Meadus RJ, Snow N, Didham P. Patient perception of contemporary nurse attire: A pilot study. Int J Nurs Pract. 2014; 20(20): 149-55. https://doi.org/10.1111/ijn.12160 